\title{
What Connects Dark Matter and Black Holes?
}

\author{
Russell Bagdoo \\ Saint-Bruno-de-Montarville, Quebec, Canada \\ Email:rbagdoo@gmail.com,rbagdoo@yahoo.ca
}

How to cite this paper: Bagdoo, R. (2020) What Connects Dark Matter and Black Holes? Journal of Modern Physics, 11, 168-195.

https://doi.org/10.4236/jmp.2020.112011

Received: November 28, 2019

Accepted: January 28, 2020

Published: January 31, 2020

Copyright $\odot 2020$ by author(s) and Scientific Research Publishing Inc. This work is licensed under the Creative Commons Attribution International License (CC BY 4.0).

http://creativecommons.org/licenses/by/4.0/

\section{(c) (i) Open Access}

\begin{abstract}
Dark matter is a major component of the universe, about six times more abundant than ordinary visible matter. We measure the effects of its mass, but it escapes the telescopes. It has the particularity of emitting no radiation and interacting only by the action of gravity. The main purpose of this article is to try to answer what dark matter is: we conjecture that it is composed of magnetically charged neutrinos, true magnetic monopoles. But that requires a huge conceptual leap: Maxwell's laws must be inverted and the electric charge becomes a magnetic charge. Asymmetric "reversed" Maxwell's laws would provide the "dark" magnetic charge that would replace the electric charge. The very form of the Dirac equation, which imposed on ordinary matter that the particle carries an electric charge and obeys the principal properties of the electron, would impose in the dark matter that the "dark" particle obeys the main properties of a neutrino associated with a magnetic charge. The second aim of the article is to show that dark matter is derived from black holes, mainly from active supermassive black holes. This requires a second conceptual leap: the horizon of the black hole undergoes a high temperature and an intense pressure of magnetic fields which cause a blackout and a phase transition (or broken symmetry) when the matter crosses the horizon. The result is a reversal of Maxwell's laws: a magnetic charge is substituted for the electric charge, and the electric current becomes a tributary of the magnetic current. A third important conceptual leap follows: sterile magnetic neutrinos created inside the black hole would cross the horizon to the outside to constitute dark matter.
\end{abstract}

\section{Keywords}

Dark Matter, Magnetic Monopole, Inverted Maxwell's Equations, Magneto-Electric, Dirac Equation, Magnetic Sterile Neutrino, Active Black Hole, Event Horizon

\section{Introduction}

The problem of dark matter is well known: observational evidence and theoreti- 
cal arguments suggest that there is a lot more matter that gravitationally interacts in the universe than what is accounted for. This is not the normal "baryonic" material in the cosmos. Yet no direct evidence exists to explain what dark matter is. This hidden form of matter must be a kind of particles which do not feel the electromagnetic or strong forces and which, consequently, neither emit nor reflect light nor behave like atomic nuclei bound together. Observations suggest that most of the dark matter is "cold".

Scientists have theorized several potential explanations of dark matter that appears to exert a gravitational pull on normal matter in galaxies and clusters of the cosmos. These possibilities belong to different categories. Some scientists suggest a complex dark matter, a wide range of dark species. Wimps, axions, heavy and sterile neutrinos, low mass black holes and dark matter atoms are among the contenders for dark matter envisioned by theorists. The simplest theories of dark matter postulate that only one type of particle contributes to the invisible mass [1].

Although cosmologists do not know what constitutes dark matter, they know something about its properties from their observations of how it influences ordinary matter and by simulations of its gravitational effects. They know that dark matter travels at a much slower speed than light, and that it therefore aggregates more than fast-moving "hot" dark matter, that it must be electrically neutral because it does not absorb or emit electromagnetic radiation. The particles that make up dark matter are probably massive. They cannot interact by the strong force that binds the atomic nuclei together; otherwise we would have had evidence in the interaction of dark matter with cosmic rays. Until recently, scientists believed that dark matter might interact via the weak force, but new observations have wiped out this notion.

In this article we conjecture a new force that belongs essentially to dark matter. It would be a magnetoelectric force (ME) that would be an inverted form of the electromagnetic force (EM). This would result in the existence of a magnetic charge that would replace the electrical charge. Maxwell's four laws would be reversed. We imagine a sterile neutrino with a magnetic charge that would emit gravitons and plays the equivalent role of the electron that emits and captures photons. What's more, these sterile neutrinos would come from black holes.

At first glance, there is no relationship between dark matter and a substance emanating from a black hole, since black holes are often defined as the regions from which nothing can escape, not even light. Stephen Hawking demonstrated, however, that there are some reasons to think that particles can come out, by "tunnel effect": the space-time in which we evolve would be modeled by the connections established between the black holes, via a purely quantum link.

In recent years, a new vision of the world has put black holes in the center of the stage. Astrophysicists believe that black holes are responsible for phenomena ranging from X-ray emissions to huge jets of material ejected from galaxy centers. The big bang would hide a primordial black hole located in another universe and from which ours would have emerged. Space-time would be woven 
with micro black holes connected together by a quantum phenomenon. Stars and galaxies are thought to have been created by jets of matter expelled by supermassive black holes. The black holes that were taken at first for the worst cosmic monsters would actually be the greatest builders [2].

The idea that black holes are dark matter has been proposed by several theorists. They first thought of massive primordial black holes formed in the first second of the universe. But it would take billions to explain the missing mass, and we would see their influence on the motion of the stars. In the 1990s, they then thought of micro black holes, of the order of a nanometer, but weighing one hundredth of the mass of the Moon. Except that their evaporation would have been detected by the gamma satellites in the 2000s. They are currently studying the possibility of primordial black holes weighing between 20 and 100 solar masses. Gravitational wave detectors have seen the fusion of objects in this category in recent years.

Our hypothesis is not that black holes are dark matter, but that dark matter would consist of substances from black holes, including sterile neutrinos associated with a magnetic charge. We say that a "black out" happens at the event horizon of a black hole (due to the enormous pressure and high temperature) that reverses the Maxwell's laws, transforms the electric charge into a magnetic charge, and makes invisible and imperceptible particle emission. Active black holes are internally filled with "dark" energy. According to the theory of Relation [3] [4], this energy would be the dark energy of the beginning (amalgamated with the kinetic negative energy, with the cosmological constant) which has dissolved to form the ordinary matter with positive energy, according to the principle of Compensation. This energy is the same as that of polarized vacuum, except that it is all the more excited as the temperature is high. The strong fluctuations cause the expulsion of sterile neutrinos inside the black hole horizon with a relativistic speed close to the speed of light. The emitted particles become slower and "magnetized" with cooling. They will automatically be magnetic monopoles.

This paper will address three links between dark matter and the black hole. These are actually three conceptual leaps. In Section 2, "Dark Matter and Maxwell's Laws Reversed", we see that dark matter might interact with a form of light to which our eyes are blind (Sect. 2.1, 2.2). It would be a variant of electromagnetism, a "magnetoelectric" force that would be obtained by reversing Maxwell's laws: this is the first conceptual leap (Sect. 2.3, 2.6, 2.7). From Dirac's theory, which establishes a connection between the smallest electrical charge and the smallest magnetic pole (charge), we end up with a magnetic pole that looks like a dark matter and would be composed of magnetic sterile neutrinos (Sect. $2.4,2.5,2.8)$. In Section 3, "Black Hole", we see that black holes now play an important role in the birth and evolution of galaxies. Throughout the universe, black holes relativistic jets condense gases and trigger outbreaks of stars (Sect. 3.1). We speculate that black holes are surrounded by magnetic walls that serve as an event horizon (Sect. 3.2). This is our second conceptual leap. These mag- 
netic walls, generated by the high temperature and the intense pressure of the magnetic fields, would disturb any object meeting them. There would be a concomitant blackout with an inversion of the laws of electromagnetism and the appearance of a magnetic charge that squeezes out the electric charge. The black hole would produce a dark substance similar to that of dark matter (Sect. 3.2.1 to 3.2.5). In Section 4, "Creation of Magnetic Sterile Neutrinos inside the Active Black Holes that can Cross to the outside to Constitute Dark Matter", we figure that the black hole space is filled with dark energy. In the theory of Relation, there was at the beginning a maximum energy (identified with dark energy) which declined by transforming itself into ordinary matter. The black hole does the opposite process by turning ordinary matter into energy (Sect. 4.1). Some will feel that this paper deals with the problem of dark energy in a manner that is not consistent with the standard model of particle physics and general relativity. We argue, on the contrary, that it is the problem of dark energy that is inconsistent with the standard model of particle physics and general relativity, and we explain why (Sect. 4.2). It is logical to expect that the gravitational energy density inside the black hole can easily convert into virtual couples of particles and materialize them. This enormous energy would behave like an intense accelerator of materialization and annihilation. "Dark" particles and antiparticles could escape from the black hole. Third conceptual leap: the sterile "magnetic" neutrinos could be the dark matter (Sect. 4.3). In Section 5, "Efforts of Four Researchers", we highlight some aspects of the work of four researchers who are contributing to the extension of knowledge about dark matter, magnetic monopoles, black holes and sterile neutrinos. In Section 6, "Heat, entropy and information have everything to do with black holes", after describing the problem of entropy (Sect. 6.1) and the information paradox (Sect. 6.2), we presume that not only information escapes from the black hole, but also the destroyed matter (Sect. 6.3). In section 7, "Comments and Conclusion", we realize that dark matter, different from ordinary matter, generates a crisis. A major conceptual overhaul is needed. It concerns, in addition to dark matter, electromagnetism, sterile neutrino and black holes. A final summary serves as conclusive.

\section{Dark Matter and Maxwell's Laws Reversed}

\subsection{Omnipresence of Dark Matter in All Regions of the Universe}

The suspicion of the existence of a dark matter is due to the astronomer Fritz Zwicky in 1930. He noticed a dynamic anomaly within each cluster of galaxies whose mass he proposed to determine by measuring the speed of galaxies constituting these clusters. The speeds of the galaxies were too great to be balanced by the gravitational pull of the cluster, which should have been scattered. He came to the conclusion that the mass of these clusters must be greater than all that was observable and that a hidden matter must be present in each cluster. He estimated that the hidden mass represents more than $90 \%$ of the mass of the cluster.

Around 1960, astrophysicist Vera Rubin, while studying the dynamic behavior 
of gaseous clouds orbiting the center of certain galaxies, discovered that this unknown dark matter was also distributed outside the clusters. These clouds are sometimes located at distances from this center that sometimes far exceed the visible radius of their galaxies and the rotational speeds of these galaxies should have decreased with their distance from the center of the galaxy. She realized that the speed of rotation of the gaseous clouds was independent of their distance from the galaxy. If all the matter, visible and dark, had been concentrated in the galaxies, the speed of rotation of these clouds should have been all the smaller as their distance in the center was great. All these experimental facts testified to the presence of dark matter, uniformly distributed not only within these galaxies, but also in vast external volumes. Observations on larger scales, clusters of galaxies that cover a few million light-years, have confirmed the presence of dark matter [5].

\subsection{The Candidates}

Theorists consider radically different paths to explain this unknown fluid of ordinary matter that bathes the whole cosmos and whose nature remains to be explained. Over the decades they have scrolled through several candidates: wimps (neutralino, Kaluza-Klein particle, little Higgs particle), wimpzilla, axions, machos, black holes, sterile neutrinos, etc.

Wimps (weakly interacting massive particles) are the preferred candidates. These hypothetical particles have in common to be more massive than the particles known today, and are supposed to be able to interact with the latter only via the force of gravity and the weak nuclear force. These are ideal candidates for dark matter, as it is assumed that they would have just the abundance required to explain the current structure of the universe. Several distinct theories, all supposed to correct the imperfections of the standard model that describes particle physics, predict different types of wimps. For twenty years, astrophysicists and particle physicists have given themselves the means to discover them. Whether it is a direct detection (the aim is to detect the impact of a wimp on a core of ordinary material in an underground laboratory) or indirect (the products of the collision of two wimps are tracked in galaxies, in the heart of the sun, in cosmic rays, in the LHC at CERN), no dark matter particles have been detected to date [6].

The wimps' track, as well as those of the other candidates, could be a dead end. So instead of looking for a new particle, why not change the law of gravitation? This is what the followers of Mond (Modified Newtonian Dynamics) have been trying to do for thirty-five years. But neither this theory nor its most recent varieties can explain all the properties of dark matter.

\subsection{A Variant of Electromagnetism}

It seems that particle physicists are living a nightmare scenario. It turns out that they found no new particles beyond the standard model with their accelerators. 
They took a first look everywhere and found nowhere. They still continue to move forward. They rely on the great diversity of detection approaches to hope to one day get their hands on the good particle(s). We believe that this crisis must be solved by a major conceptual overhaul. Without being guided by theoretical prejudices, we propose a variant of electromagnetism that will provide an explanation for dark matter. Why would something remarkable and unprecedented not have occurred at the heart of the electromagnetic theory that would make it incapable of emitting or absorbing electromagnetic radiation?

There is almost complete symmetry between electrical and magnetic phenomena. The difference lies in the fact that no free magnetic pole exists (north or south), while there are free electric charges (positive or negative): the two types of poles can never be physically separated. This makes us consider magnetism as a secondary phenomenon whose existence depends on the flow of an electric current [7].

Maxwell's four equations fully describe the electromagnetic behavior on a very large scale, including that of light. The electromagnetic field is the space between the lines of force of the electric field $E$ and the magnetic field $B$, and there is thus energy

$$
U=(\varepsilon / 8 \pi) E^{2}+(\mu / 8 \pi) B^{2} .
$$

( $\varepsilon$ : vacuum permittivity; $\mu$ : vacuum permeability)

Suppose a severe astrophysical event has occurred that would cause the visible light to cease. And that to provoke this darkness, it would have been necessary that the electric charge no longer plays its role, that its physical size becomes another (the letter $q$, in Coulomb's formula, would no longer play the exact role played by the letter $m$ in the Newton's formula). This last possibility has already been considered by Paul Dirac, while he was wondering about the reason for the existence of the smallest electric charge.

\subsection{Dirac's Theory Establishes a Connection between the Smallest Electrical Charge and the Smallest Magnetic Pole}

Although in classical electromagnetism the existence of magnetic monopoles is not compatible with Maxwell's equations, and although special relativity allows us to demonstrate all Maxwell's laws, including that which predicts the non-existence of magnetic monopoles, Paul Dirac demonstrated in 1931 that the existence of magnetic monopoles was compatible with Maxwell's equations in the hypothesis of the quantification of the electrical charge [8]. His theory establishes a connection between the elemental electric charge (that of the electron) and the hypothetical elementary magnetic charge. It showed symmetry between electricity and magnetism, which is still completely foreign to established conceptions.

We know that the smallest electric charge exists experimentally. With a purely electronic quantum condition, we obtain the value $e$ (in CGS system) given approximately by 


$$
\begin{gathered}
\hbar c / e^{2}=137.03 \\
\left(1.054512 \times 10^{-27} \mathrm{erg} / \mathrm{sec}\right)\left(2.9979 \times 10^{10} \mathrm{~cm} / \mathrm{s}\right) /\left(4.8030 \times 10^{-10} \text { statcoul }\right)^{2}=137.03
\end{gathered}
$$

However, his theory, although at first intended to give a theoretical value to e, turned out, when it was developed, to establish a connection between the smallest electric charge and the smallest magnetic pole, namely the equation

$$
\begin{gathered}
\hbar c /\left(e \mu_{o}\right)=2 . \\
\left(1.054512 \times 10^{-27}\right)\left(2.9979 \times 10^{10}\right) /\left[\left(4.803 \times 10^{-10}\right)\left(3.29028 \times 10^{-8}\right)\right]=2
\end{gathered}
$$

( $\mu_{o}=3.29098 \times 10^{-8}$ statcoul : quantum of magnetic pole). Although Dirac's proof of the relation between pole strength, electric charge, and Planck's constant, is not simple, we can, by a simple estimate, explain the number 2 of the Equation (3) and briefly illustrate the character of the relation [9]. Here we consider that poles exist and one isolated plate (a capacitor plate holding a magnetic pole charge) holds a pole density of $\sigma_{\mu_{o}}$ poles per unit area. By analogy with the calculation of the electric field from such a plate holding an electric charge density and the symmetry between the electric field $E$ and the magnetic $B c$, we find that the magnetic field near the plate is

$$
c B=2 \pi \sigma_{\mu_{o}} .
$$

The electric charge moving with a velocity $v$ will move in a circle of radius $r$ if the centrifugal force on the charged particle, $m v^{2} / r$, is equal to the force on the moving charge generated by the magnetic field, $\mathrm{Bev}$

$$
B e v=\frac{m v^{2}}{r} \text { or } B e=\frac{m v r}{r^{2}} .
$$

From the quantization of angular momentum, $m v r$ is equal to $n \hbar$, where $n$ is an integer. Substituting the pole density expression for $B\left(B=2 \pi \sigma_{\mu_{0}} / c\right)$,

$$
\frac{2 \pi \sigma_{\mu_{o}}}{c} \cdot e=\frac{n \hbar}{r^{2}} \text { or } 2\left(\pi r^{2} \sigma_{\mu_{o}}\right) \cdot e=n \hbar c .
$$

This relation requires that the magnetic pole strength enclosed by the orbit of the electron be quantized. Setting that pole strength to be the smallest value, one single pole with a unit pole strength $\mu_{o}$, we have $\pi r^{2} \sigma_{\mu_{o}}=\mu_{o}$ and

$$
\mu_{o} \cdot e=\frac{n}{2} \hbar c \text { hence } \hbar c /\left(e \mu_{o}\right)=2 \text {. }
$$

Although this estimate is crude, it serves to illustrate the connection between the quantization of angular momentum and the quantization of charge and pole strength. Quantum mechanics requires a quantization of charge-if monopoles exist.

Instead of finding a purely electronic quantum condition, such as (2), Dirac found a reciprocity between electricity and magnetism, a connection between the magnetic pole quantum and the electronic charge. His theory contains no arbitrary characteristic, gives no possibility to modify it, and would have the ef- 
fect of creating a magnetic monopole. For that, the theory requires a quantization of the electric charge, because any charged particle moving in the field of a pole of intensity $\mu_{o}$ must have as it charge an integer multiple (positive or negative) of $e$, so that functions wave describing the motion may exist.

Magnetic poles would have properties similar to those of electric charges. Each pole would emit $4 \pi \mu_{o}$ of magnetic field lines $B$, where $\mu_{o}$ is the pole strength (corresponding to the charge $q$ ). The strength on a pole in an electromagnetic field would be

$$
F=B \mu_{o}+E \mu_{o} \frac{v}{c},
$$

where, field vectors and velocity are orthogonal. The strength exerted on a fixed magnetic pole would be a measure of the magnetic field and the strength exerted on a moving pole would be proportional to the pole's speed and the magnitude of the $E$ field. The symmetry of the pole and the charge seems to be exact.

If the charges and poles are so similar, why hasn't nature provided us with poles? (Poles have not been seen despite careful searches.) However, if poles are found they must have much larger charges than the unit electrical charges found on elementary particles such as the electron. So this universe cannot be completely symmetric between pole and charge on the microscopic level.

We might still ask why, if poles and charges are symmetric in principle, we have charges and not the poles. If the universe were constructed so that there was no electric charge, but only magnetic poles with the same value of pole strength as the fundamental charge strength, we believe that this universe would be indistinguishable from ours. If we could communicate with the inhabitants of that universe (who are made of protons, which have no electric charge, but hold a unit magnetic pole strength, and electrons, with no charge, but with an opposite magnetic pole strength), we could not determine whether they live in a magnetic universe or an electric universe as we do [9].

\subsection{Our Theory for Dark Matter: Sterile Particles Associated with Magnetic Monopoles}

And if the universe was constructed in such a way that there is no electrical charge, but only magnetic poles not having the same value of pole strength as the fundamental charge strength, so that the left-hand side of Equations (2) or (3) no longer corresponds to the experimental value 137 or the theoretical value 2 , we think we would be in a total darkness that would have the appearance of a dark matter. We can imagine that the elements that make up this dark matter would be composed of elements charged magnetically, with electricity and the electric field considered as a relativistic consequence of the magnetic field, which involves reversing Maxwell's laws.

\subsection{Inversion of Maxwell's Laws}

The experimental dissymmetry of Maxwell's equations with respect to the elec- 
tric-magnetic duality is related to the fact that the electric field is generated by the usual charges which give it a non-zero divergence, but the magnetic field is always of zero divergence because of the absence of corresponding punctual charge. Experimentally, the only source of the magnetic field comes from the existence of an electric current, that is to say a motion of electric charges.

$$
\begin{gathered}
\nabla \cdot E=4 \pi \rho_{e}, \\
\nabla \cdot B=0, \\
\nabla \times E+1 \partial B / c \partial t=0, \\
\nabla \times B-1 \partial E / c \partial t=4 \pi J_{e} / c .
\end{gathered}
$$

( $\rho_{e}$ : electric charge density; $J_{e}$ : electric current density; $\sigma_{\mu_{o}}$ : magnetic charge density; $J_{\mu_{0}}$ : magnetic current density; e: electric charge; $\mu_{o}$ : magnetic charge) [10].

Assuming there is a magnetic charge density $\sigma_{\mu_{o}}$ and a current density $J_{\mu_{o}}$ but no corresponding electrical counterpart, the equations would be asymmetric being fully subject to the magnetic charge. Maxwell's equations then become:

$$
\begin{gathered}
\nabla \cdot B=4 \pi \sigma_{\mu_{o}}, \\
\nabla \cdot E=0, \\
\nabla \times B+1 \partial E / c \partial t=0, \\
\nabla \times E-1 \partial B / c \partial t=4 \pi J_{\mu_{o}} / c .
\end{gathered}
$$

The equations are still asymmetrical but no longer subject to the electric charge. Equations (14) and (15) seem to miss something on their right sides. To see exactly what they are missing, we need to explain the meaning of $\nabla \cdot E$, also called divergence of $E$ or simply of $\operatorname{div} E$. Let $V$ be a volume surrounded by a surface $S$ in space. $\nabla \cdot E$ integrated on the volume $V$ gives $4 \pi$ times the total amount of electric charge $e$ contained in $V$. Similarly, $\nabla \cdot E$ evaluated at point $x$ gives $4 \pi$ times the electric charge density at $x$. Hence, Equation (14) indicates that there is no electric charge at any point in space. Basically, moving charges are equivalent to currents. But because the above reversed Maxwell's equations assume that there is no electric charge in dark matter, there is no electric current $J_{e}$ on the right side of Equation (15). Equations (13) and (16) seem to have won something on their right sides. This means that $\nabla \cdot B$ integrated on the volume $V$ surrounded by a surface $S$ in space gives $4 \pi$ times the total quantity of magnetic charge $\mu_{o}$ contained in $V$. Similarly, $\nabla \cdot B$ evaluated at the point $x$ gives $4 \pi$ times the density of magnetic charge at point $x$. As a result, Equation (13) indicates that there is a magnetic charge at any point in space.

Because the Maxwell's equations above assume that there is a magnetic charge, there is a magnetic current $J_{\mu_{0}}$ on the right side of Equation (16). Therefore, the absence of electric charge and the presence of magnetic charge reverse the asymmetry.

In fact, the electrical charge would become a magnetic pole, which would result in an attribution reversal, so that electricity should be considered as a sec- 
ondary phenomenon whose existence depends on the flow of a magnetic current. The overthrow, in addition to the darkness caused, would in a way make that there would be free magnetic poles when there would be no more free electric charges. Magnetic monopoles would exchange "dark photons".

Note: There is no question of continuing by presenting a critical analysis of the hypothesis of Maxwell's "inverted" laws, because these must not be considered in an absolute sense, as if the nature of dark matter had to conform precisely to these laws. It is only a simplistic schema of reality, a kind of approximation, an image. As such, it corresponds to reality, even if it does not identify with reality.

\subsection{Magnetoelectric Force}

The inversion that we have just presented shows that there would be a dark magnetoelectric force (ME) with a dark photonic wave, just as there is an electromagnetic force (EM) with a photonic wave. Dirac's theory ensures that the magnetic monopole can coexist with an electric charge in ordinary matter. Maxwell's laws are not reversed, they are completed in order to obtain a perfect symmetry: $E \neq 0, B \neq 0$. According to our hypothesis of the inversion of the laws of Maxwell, the magnetic pole would replace the electric charge: $E=0$, $B \neq 0$. There would only be a magnetic pole, which evicted the electrical charge.

We suggest the existence of an electric charge (known electric monopole) in ordinary matter and a magnetic pole (unofficial lightweight magnetic monopole) in dark matter. With rare exceptions, there is no coexistence of the two charges in ordinary matter or in dark matter. There would be no electric monopole in dark matter just as there would apparently be no magnetic monopole in ordinary matter.

\section{8. "Magnetic" Sterile Neutrinos}

To penetrate the mystery of dark matter, we think that it is a different electromagnetism, a magnetoelectrology, with the necessity of qualifying this variant as a "new force". And that it is also a new particle: "magnetic" sterile neutrino.

Physicists know three types of neutrinos. Since the 1970s, many researchers have assumed that there is a fourth type, a "sterile" neutrino, much heavier, but which interacts even less than the others with ordinary matter. It is a hypothetical type of neutrino that does not interact via any of the fundamental interactions of the standard model of particle physics except gravity. It is a right chirality neutrino or a left chirality antineutrino that can be added to the standard model and can take part in phenomena such as the mixing of neutrinos.

We assume that there is a fifth type of neutrino. Our hypothesis is that there would be a sterile neutrino linked to magnetic pole, that would belong to dark matter and that would be a magnetic monopole. The term magnetic sterile neutrino is used to distinguish it from sterile neutrino. The mass of the neutrino in both cases is unknown and could take any value between less than $1 \mathrm{eV}$ and $10^{15}$ 
$\mathrm{GeV}$.

Dark matter would consist of invisible magnetic sterile neutrinos that swarm in the universe and exert a gravitational attraction everywhere [11]. This sterile neutrino would depend on a magnetic pole that would be undetectable since it is not an integral multiple of the conventional electric charge. Where would it come from? Our idea is that they come from active black holes, which risks upsetting our vision of black holes even more.

\section{Black Holes}

\subsection{Black Holes Revolution outside the Event Horizon}

Black holes are giving birth to a new genesis of the universe at every level. Our universe would have emerged from a primordial black hole that would be the big crunch of another universe. Initially, the black holes were at first only a mathematical singularity, a cosmic curiosity very difficult to observe. These gravity wells come from general relativity, they are an effect of the existence of a spacetime curved by the masses and it is only in 1968 that John Wheeler invents the expression "black hole". As early as 1916, Karl Schwarzschild found the solution of general relativity that describes the gravitational field around a star. A singularity in its heart is seen as impenetrable before becoming, after the 1970s, the horizon of the black hole that swallows everything. From the 1990s, with the progress of observations, there is a need to believe that they really exist. Astronomers build a bestiary of destructive black holes. There would be everywhere: in the primordial universe, at the center of our Milky Way and all galaxies. Cosmologists and physicists see the concept of the black hole as a means to marry the irreconcilable theories of relativity and quantum physics. The proof of their existence fell on September 14, 2015 when the Ligo experiment for the first time captured gravitational waves caused by the fusion between two black holes.

Today, it seems that those who were thought to be the worst cosmic gluttons have become the great architects of the universe. They would have structured the primordial universe, modeled galaxies and lit up stars [2]. Supermassive black holes have been observed everywhere in the cosmos and astrophysicists believe that one of them sits at the center of most galaxies. They concentrate millions of times the mass of the Sun and would have created stars and galaxies. Observations made in the late 1990s were the first to reveal three creative roles of supermassive black holes:

\section{First, a role of regulator, guardian of galaxies.}

Astrophysicists first realized that in nearby galaxies, the central black hole always seemed to weigh $1 / 1000$ of the star bulb that shelters it, a sign that the two are linked. Then, from 2005, it became apparent that the energy of the most energetic black holes can modulate star formation, stopping the formation of galaxies that otherwise would be enormous. Plasma winds ejected by the disk from black holes could control the growth of galaxies. These winds reach 1/10 the speed of light and carry enormous amounts of energy. In this way, today, 
$80 \%$ of the gas in the universe is found outside galaxies. Thus, the black holes play a regulatory role, soothing through their winds a cosmos too eager to generate stars.

\section{Second, a role of cleaner (ionizer) of the primordial universe.}

They could also, in other circumstances, have played the opposite role: concentrating the gas clouds, boosting the star formation, they would have been the main actors of the reionization process some 400,000 years after the big bang. Their X-rays, much more energetic than the UV of stars, could have been extracted from galaxies and ionized the intergalactic medium at greater distances than the UV of stars. Even smaller black holes, the stellar black holes a few dozen times the mass of the Sun, could have participated because in this primordial universe, they were always accompanied by a star whose matter they vampirized. What to maintain over the long term their production of X-ray at a high rate. They could thus disperse the thick neutral hydrogen fog by ionization of the atoms and make the cosmos transparent.

Third, a trigger role of star births through their jets.

A supermassive black hole compresses and heats the gaseous material that accumulates and revolves around it so much that this burning plasma begins to radiate and create an intense magnetic field. The radiation pressure exceeds gravity. Strong winds are generated in all directions and some of the matter escapes from the poles, in the form of two fine jets, several hundred $\mathrm{km} / \mathrm{s}$ away. The winds blow the galaxy's gas and regulate its star production. The jets strike distant clouds of gas, initiating their condensation into new stars. This is suggested by the observation of some active galaxies. It seems proven, that with their jets, black holes form stars. It was discovered that a surge of new stars followed the direction of the jet emitted by its central black hole. The jet is so powerful that in a short time it can form $10 \%$ of a galaxy, like a spider spinning its web. Although there are only a handful of examples, black holes are now taken into account in the theory of galaxy formations.

\subsection{Black Holes Revolution inside the Event Horizon}

The three previous roles involve the photon sphere and the accretion disk outside the event horizon. We propose here a fourth role, a role of creator of dark matter, by their emission of "magnetic" sterile neutrinos. This role concerns the internal space of the black hole, between the horizon and the center of the black hole.

\subsubsection{The Classic Horizon of the Black Hole}

Roger Penrose, wrote a short article in 1964 in the journal Physical Review Letters, where he described the problem of the singularities associated with star implosions and demonstrated a mathematical theorem that said that when a star collapses to the point where gravity becomes strong enough to form an apparent horizon around it that brings back the photons that are trying to emerge, nothing can prevent the gravity from becoming strong enough to create a singularity. 
Therefore, any black hole must contain a singularity. In the late 1960s, Penrose searched unsuccessfully for a mathematical example of a collapse that produced a naked singularity. In 1969, he issued the conjuncture of cosmic censorship: no object can, collapsing, give birth to a naked singularity. If a singularity is formed, it is dressed with a horizon that makes it invisible from the outside world [12].

This apparent horizon (like a spherical membrane) is in fact the Schwarzschild horizon. It is not singular in the strong sense, space-time is defined and it is permeable to incoming particles, it is a unidirectional membrane. The membrane of a sphere formed of light rays which define its surface. At the center of Schwarzschild's solution lies the true singularity, the heart of the black hole. The Schwarzschild sphere is an apparent singularity called horizon $\left(r=2 G M / c^{2}\right)$, while the point of space-time at the origin of coordinates $(r=0)$ is really singular and looks like what we call the big bang [13].

At the crossing of the horizon, time-coordinate $t$ and radius- coordinate $r$ have exchanged their roles because the sign assigned to them in the definition of the linear element of space-time turns over. Which means that time becomes space, and space, time. The time-coordinate $t$ is not at all adapted to the local definition of $d s^{2}$ since we no longer recognize the signs of time and space. In other words, the Euclidean metric does not extend inside the horizon.

But if we take a quantum view of cosmic censorship, the collapse of the structure at the level of a singularity must not affect any physical measurement. A description of the particle in free fall should allow to drive the particle through the horizon to the center by a path integral [14].

The most general model of black holes, according to general relativity, says that imploding stars towards the state of the black hole must, by passing their horizon, lose all the differences to the spherical symmetry, "all their hair", all their characteristics (except three parameters: mass, charge, angular momentum), and therefore, for example, their protuberances, their asymmetries and their magnetic field; they must, willingly or forcibly, become "bald". This lost structure must be evacuated previously in the form of radiation, in the form of an emission of gravitational waves.

\subsubsection{Power Failure at the Crossing of the Horizon}

It may be said that this model predicts the emission of gravitational waves, but the particle of the collapsing star does not end its life on the horizon, as if it were finally dying in the center, on the true singularity. At the crossing of the horizon, it seems to simply disappear on one side to reappear the other side. It becomes invisible to any observer left outside the sphere. Everything happens as if the sudden invisibility of the light was caused by a blackout that begins on the horizon. How to understand what happens to a particle, whether material or luminous, immediately after it has crossed the horizon? One could perhaps understand by taking an ordered magnetic field that would have settled on the "enlarged horizon" of a black hole located in the center of a quasar. An enlarged horizon is a fictional area just outside the horizon while an "inner horizon" is a fic- 
tional area just inside the horizon. This black hole is surrounded by an accretion disk, composed of hot and ionized gases. When plasma detaches from the inner edge of the disc and plunges onto the enlarged horizon, it carries with it a skein of magnetic field lines. When this magnetic field crosses the enlarged horizon, it generates surface currents that dissipate energy by flowing into the very resistive membrane. In fact, the lines of the field do not cross the real horizon, but wrap around it and form loops. The density of these loops and the intensity of gravity are such that they cause symmetry breaking in the membrane. There is a charge reversal; the magnetic pole replaces the electric charge. It's the blackout.

\subsubsection{Magnetic Field of the Horizon and Energy of the Quasars}

To provide the energy of a quasar, a magnetic field should cross the enlarged horizon throughout the life of the quasar. However, there is a source, outside the black hole, likely to generate such a field: the interstellar gas attracted to the black hole. The interstellar gases are the seat of magnetic fields and, when the gases heat up and ionize near a black hole, they form a plasma disk where the field lines are "frozen". The rotation and turbulence of this plasma in accretion entangle the field lines, some of which settle on the enlarged horizon, during the fall of plasma fragments. In the membrane, surface currents continuously dissipate the energy of this chaotic field, leaving only "clean", ordered, field lines that penetrate the membrane at the South Pole and exit at the North Pole. After an ordered field line has been deposited on the black hole, it no longer disappears: the plasma of the accretion disk and the magnetic field make it persist as long as the disk does not explode or is not swallowed by the black hole. The black hole acquires a magnetic field more than 10,000 times more intense than the Earth's magnetic field.

\subsubsection{Where Electromagnetism Becomes Magnetoelectricity}

It is known that the sphere of the event horizon is surrounded on the outside of a sphere of photons where, because of the considerable gravity, the particles of light no longer propagate in a straight line but begin to orbit. These spheres are surrounded by an accretion disk which is a plasma disc formed of matter taken by the attraction of black holes, compressed under the effect of gravity and heated to thousands of billions of degrees. The inner edge of the accretion disk is the last place where matter can orbit before falling into the black hole. A supermassive black hole compresses and heats the matter around it so much that this hot plasma creates an intense magnetic field. The latter exerts on the horizon of the black hole forces perpendicular to itself. The magnetic tension force is inversely proportional to the radius of curvature of the field line: it acts by stretching the field lines as if they were elastic cords. The behavior of the field can be interpreted as if it were endowed with a throttling pressure: when the forces of the magnetic field penetrate inside the horizon of the black hole there is a phase transition where the relation electricity/magnetism is changed due to too high pressure and excessive temperature. There is a charge reversal, during which the 
electric charge disappears to become the magnetic pole. We go from light to darkness; we witness a kind of reversal of Maxwell's laws, as described above [15].

With the reversal of charges, electric currents become electric fields and magnetic fields become magnetic currents. The result is that on the horizon, this region of space-time pressed by the electromagnetic tidal forces which culminate, the light does not act suddenly anymore. To illustrate the phase transition, consider that electromagnetism, before breaking through the horizon, is like a piece of wood impregnated with water. In this analogy, wood is magnetism and water is electricity, and both (wood and water; magnetism and electricity) are intimately intertwined, unified. Close to the horizon, according to our better understanding, the laws of quantum mechanics begin to combine with those of Einstein's general relativity and are already beginning to change the "rules of the game". (They will be totally changed at the singularity, and the new rules will be called quantum gravity.) The horizon and the laws of gravity combined with those of the quantum mechanics that govern it are like a fire in which wood swollen with water is thrown. The fire boils the water coming out of the wood, leaving it alone and master. On the horizon, the laws of quantum gravity expel electricity, leaving magnetism alone and resistant. Electricity is reduced to a current without conduction, extinguished [12].

One could also explain what happens with invariance groups that have proven themselves in quantum mechanics. They are algebraic transformations that retain the form of the equations and reveal physical properties. However, if we imagine a particle that crosses the horizon of the black hole, it "oscillates", it has a charge no longer electric but magnetic: it is the magnetic monopole. The particle described by the Dirac equation thus acquires another gauge invariance: an inverted gauge invariance. The Dirac equation might have a gauge invariance that changes a bit the wave described by the equation, but the new particle does not just change "phase", it is no longer an integral multiple of the charge of the electron. It no longer interacts with electromagnetism. Nevertheless, there are other "dark" invariance groups that fall under "magnetoelectrology" and that can intervene in accessible disintegration phenomena because these monopoles are not only magnetic but endowed with weak interactions.

To summarize, on the periphery of the black hole, there is a horizon: a region where light no longer works and where electromagnetism has given way to magnetoelectricity. This means that with the reversal of charges, electric currents become electric fields and magnetic fields become magnetic currents. In a pictured language, it will be said that electric fields are "frozen" in magnetic currents.

\subsubsection{Light Can Escape from the Black Hole}

In the context of general relativity, a black hole is defined as a gravitational singularity occulted by a horizon of events. It is a celestial object so compact that the intensity of its gravitational field prevents any form of matter or radiation 
from escaping it. According to quantum physics, a black hole is likely to evaporate by the emission of black body radiation called Hawking radiation. In 1974, Stephen Hawking discovered that, contrary to classical mechanics, black holes could radiate near heat radiation. The "temperature" of the black hole, which is inversely proportional to its size, is associated with it. In an article published in 2014, Hawking declared that there is no black hole, in the sense that light cannot escape to infinity [16]. According to him, these space ogres, capable of devouring galaxies and making light disappear, could actually release some quantities of matter and particles. The matter and the energy could actually be held temporarily, then modified, before being released into space. A phenomenon that would be inversely proportional to the mass of these objects: the smaller a black hole, the more it would let large quantities of matter escape. Black holes would not be so "black" as most cosmological models portray.

\section{Creation of Magnetic Sterile Neutrinos inside the Active Black Holes That Can Cross to the Outside to Constitute Dark Matter}

\subsection{Dark Energy inside the Black Hole}

The deep meaning of the discovery of Hawking radiation emanating from black holes is that the quantum vacuum is polarized by the very intense gravitational field prevailing in the vicinity of a mini black hole; the gravitational energy of the latter is converted spontaneously into particles. Quantum vacuum means minimal energy. According to the theory of Relation [3] [4] [17], there was at the beginning a maximum energy (identified with dark energy) which declined by transforming itself into ordinary matter. Black hole does the opposite process by turning ordinary matter into energy. This enormous energy is like a vacuum energy (which has become very dense) inside the black hole since it is no longer materialized. It is logical to expect that the enormous density of gravitational energy (which has a colossal mass) inside the black hole can easily convert into virtual couples of particles and materialize them.

Following the reversal of the charges explained above which converts visible ordinary matter into invisible matter, let us imagine a distribution of dark matter inside a black hole whose mass increases by engulfing a whole astrophysical jumble of gas pockets, stars, etc. As the mass of the black hole enhances, the dark matter sees its distribution contract, become more compact and denser. The black hole accumulates a colossal black mass that is equivalent to dark energy. The latter means a gigantic density of matter- dark energy inside the horizon. This enormous energy would behave like an intense acceleration of annihilation [18].

The boost in the mass of black holes thus augments the rate of annihilation of the dark energy-matter inside the horizon. In principle, because the density of dark matter is prodigiously high (inside the supermassive black holes, and even the intermediate-mass black holes of with a mass between a hundred and a mil- 
lion times the mass of the Sun), the probability exists that the rate of annihilation of particles of dark matter will accelerate to the point of injecting outwardly, out of the horizon, ample energy to constitute the unknown substance known as "dark" which fills the universe.

To describe the states of a magnetoelectric field, we will use an intra-horizon space, which is a first circle once crossed the horizon superimposed on several circles leading to the central point, called a singularity. In this intra-horizon space, the magnetoelectric field contains a huge concentration of energy, allowing large fluctuations of the creative energy of particles.

The inside of the active black hole could be comparable to the state of the universe during the 380,000 after the big bang, while the universe was a sea of darkness-just a fog of hydrogen atoms forged by the big bang and left to float in the absence of light. However, if it is constructed in such a way that there are only magnetic poles with a pole strength value other than the fundamental electrical charge strength, the inside of a black hole would be different from the inside our primeval universe. This inside could be made of a particle (similar to proton) holding a unit magnetic pole strength and a particle (similar to electron) with an opposite magnetic pole strength. These poles would provide a source of magnetic field just as an electric charge provides a source of electric field. The energy coursing through the black hole is so strong that magnetic monopoles, characterized by equal magnetic poles of similar or opposite signs, which repel or attract each other, cannot assemble a magnetic dipole.

\subsection{The Idea of Dark Energy}

We have just seen that according to the theory of Relation there was at the beginning a maximum energy (identified with dark energy) which declined while being transformed into ordinary matter. Some will feel that this paper deals with the problem of dark energy in a way that is not consistent with the standard model of particle physics and general relativity. A big nuance to bring: it is the problem of dark energy which is inconsistent with the standard model of particle physics and general relativity. Let's take a closer look.

According to official cosmologists, $70 \%$ of the contents of the universe are made up of a mysterious, undetectable and anti-gravitational dark energy that accelerates the expansion of the universe. It was through the observation of distant supernovae, which constitute "standard candles" intended to measure the universe on a large scale that they were able to deduce that dark energy existed. The latter was not predicted by any theory. It was introduced as a simple parameter in the equations of quantum particle physics and general relativity, which are two diametrically opposed theories. The result is that the dark energy, which looks like the energy of the quantum vacuum, seems to be $10^{120}$ times too strong compared to what the observations indicate. This gigantic gap is at the heart of the greatest crisis in contemporary physics.

In our opinion, we have reached this gigantic gap, or rather this unacceptable 
error, when astronomers, to measure the distance of very distant supernovae, have assumed that the intrinsic luminosity of the supernovae is the same for all, independent of the particular object measured. With this gratuitous hypothesis, impossible to prove, they came to the conclusion that expansion accelerates instead of slowing down (slowing down is what it does in an honest Friedmann model, and this is what is predicted in the equation of the theory of Relation). They then thought it wise to use an engine of unknown origin to produce the desired effect: dark energy [19].

Astrophysicists have associated this dark energy with negative pressure on a cosmological scale that would translate into a "current acceleration" in the expansion of space. It corresponds to a quantum vacuum energy whose value would be disproportionately greater. They gave this energy density value of the vacuum the same status as a repulsive cosmological constant, which pushed them to rehabilitate Einstein's cosmological constant, but about $10^{120}$ times larger. There is a deep contradiction between the concepts of quantum field theory (according to which the energy density of vacuum is about $10^{120}$ times the density of matter-energy of the present universe), and the ideas of general relativity (vacuum energy is a source of gravitation, hence of curvature of space-time) used to associate this estimate with astrophysical observations. This dark energy in the form of a repulsive cosmological constant imposed by the omnipresent quantum vacuum would produce hallucinating cosmological effects: our universe would bend so intensely that the visibility horizon would be at centimeter distances [5].

By decreeing that the supernovae of yesteryear were the same as those of today, by affirming dogmatically that the first supernovae were necessarily of a similar chemical composition of the following [20], we are arrived at the "vacuum catastrophe" or the problem of the cosmological constant. The high degree of intoxication of the scientific community was manifested by the award in 2011 of the Nobel Prize in physics to three astrophysicists belonging to two different teams for their discovery of the acceleration of the expansion of the universe. This discovery, based on the unconfirmed hypothesis of the uniformity of supernovae and uncertain distance measurements, endorsed by the judgment's passivity of official cosmology, is as aberrant as the Ptolemy's epicycles.

More and more, specialists advance the hypothesis that the acceleration of the expansion of the universe, which motivated the creation of the concept of dark energy, could in fact result from an observational bias [21]. What will be said in astrophysical publications (whose content was sadly uniform) the day when they will have to announce the non-uniformity of the concerned supernovae?

\subsection{Creation and Emission of Sterile Neutrinos in Black Holes}

Sterile neutrinos have often been proposed as dark matter candidates. It is also our preference. They would interact only by gravity with ordinary matter, with the exception of a small ability to mix with familiar neutrinos of the standard 
model. Sterile neutrinos associated with a magnetic charge would be one of the only by-products of annihilations that would successfully leak from the inside of the black hole to the outside, as would solar neutrinos associated with an electrical charge (electron) are the only ones who manage to escape from the heart of the Sun.

We will therefore limit ourselves here to the creation of sterile neutrinos dependent on a magnetic charge. The wave-corpuscle duality of photons, extended by de Broglie to the waves of matter, led to the quantum concept of matter field. This quantum field of matter is a set of operators, creations and annihilation of fermions, including the neutrino: the operator $v_{k}^{+}$creates a neutrino of pulse $k$, and the operator $v_{k}$ annihilates a neutrino of pulse $k$.

In this intra-horizon space of the active (hot and dense) black hole, which is part of Dirac's restless ocean, virtual pairs are constantly being created and destroyed. For a brief moment, a particle and its antiparticle separate. There are four possibilities

Process 1: The two partners meet and annihilate each other.

Process 2: The antineutrino remains in the black hole and the neutrino materializes in the outside world.

Process 3: the neutrino remains in the black hole and its antineutrino escapes into the outside world.

Process 4: Both partners stay in the black hole.

Particles escaping to the outside would be fermionic monopoles (refusing to put themselves in the same state). They would leave with a relativistic speed. Could there be several types of sterile magnetic neutrinos that can oscillate between them? Could the magnetic charge neutrino get the flavor of an electronic neutrino? Certainly not by an oscillation process, since all the neutrinos involved should be associated with the same type of charge. It is however possible to envisage that the sterile magnetic neutrino can decay into gamma rays (photons), into standard neutrinos (electric charge), into weaker sterile magnetic neutrinos (magnetic charge), and other particles. To return to the neutrinos escaped from the black hole, they would rather tend to slow down and regroup with the cooling to form the dark cosmos whose rules do not reflect our bright world. They would obey other non-symmetry laws, Maxwell's inverse laws, and be provided with magnetoelectric and weak interactions (very small compared to nuclear forces). The mass of these neutrinos affiliated with the magnetic charge would be rather small instead of being huge or zero, but sufficient to fill the missing mass gap.

Scientists think that there may be more than just a type of dark matter. A possibility is that several classes of dark matter particles exist, as well as a variety of forces that act only on them. One idea is that particles of dark matter interact with each other by a force that ordinary matter cannot feel. These particles could carry a "dark charge" that attracts or repels them even if they are electrically neutral and could emit "dark photons". Dark atoms would emit dark photons at 
a different rate than ordinary matter that emits ordinary photons. We know by observing the shapes of galaxies that this rhythm must be very weak.

\section{Efforts of Four Researchers}

In line with our paper, we highlight certain aspects of the work of four tenacious researchers who contribute to the extension of knowledge on dark matter, magnetic monopoles, black holes and sterile neutrinos:

Georges Lochak, president of the Louis-de-Broglie Foundation, is known for his work on magnetic monopoles: the magnetic monopole is a fermion endowed with weak interactions. He found an equation, analogous to that of Dirac, which no longer represents an electron but a magnetic monopole, which is, in a way, the other side of the electron. His equation finds Dirac's formula which shows that the charge of a magnetic monopole is equal to an integer multiple of the charge of the electron multiplied by 68.5: its equation joins this result. For him, it indicates that if the multiple is equal to zero-so if the monopole has no charge and is neutral-its equations coincide with those of the neutrino [22]. Georges Lochak worked for ten years with Leonid Urutskoiev of the Kurtchatov Institute who had headed a team that was looking for the origin of the Chernobyl disaster. Urutskoiev had hypothesized a flood of monopoles, resulting from an electrical explosion that occurred in the engine room. Some clues made him lean towards the hypothesis of a light magnetic monopole that corresponded to the Lochak monopole. Dozens of physicists contributed to a joint research work. The experiments were counted in the hundreds. The main theoretical center was the Louis de Broglie Foundation.

André Michaud explored the foundations of an electromagnetic mechanics of elementary particles whose laws apply to all levels. He described a space-time geometry that represents the mutual induction of electrical energy and magnetic energy within moving elementary particles in accordance with Maxwell's equations [23]. He details an experiment he performed that proves out of any doubt the inverse cube relation with distance between the magnetic fields of a magnet whose both north and south poles physically coincide, proving by the fact that the same inverse cube interaction law also applies by similarity to the elementary electromagnetic particles colliding with quasi-punctual behavior. This experiment also demonstrates that the magnet used behaves like a magnetic monopole [24].

Eue Jin Jeong basically demonstrated that the black hole jets and the dark matter problems are essentially one integrated physical phenomenon caused by dipole gravity. His outstanding discovery of the long ranged dipole gravity is in the fulfillment of Einstein's general relativity in its simplicity of the equivalence principle. He explains the dark matter problem in his book by invoking the fact that jets from both the south and the north poles of the rotating black hole constitutes a point source of the continuous outgoing matter following the dipole gravity force lines [25]. Jeong started early in 1982 when he was a graduate stu- 
dent, wondering why general relativity does not explain the jet phenomena from the black hole accretion discs. He was perplexed by the dismissed dipole gravity in the weak field limit of general relativity. His quest for the solution to the problem led him to realize in 1995 that the rotating hemisphere has a rotation frequency which depends on the relativistic shift of the center of mass. By investigating further, he derived Lense-Thirring force from the dipole gravity potential generated by the two hemispheres oppositely superposed inside the rotating sphere. The result is described with detailed mathematical derivation in an article published in 1999 [26].

Kevork Abazajian, an American physicist who works at the University of California, has demonstrated in an article the mechanism by which $7 \mathrm{keV}$ sterile neutrinos can be produced and be the source of unknown gamma rays observed at $3.5 \mathrm{keV}$ from center of galaxy cluster [27] [28]. Several teams of astrophysicists have observed an X-ray (gamma) spectral line with energy of about $3.5 \mathrm{keV}$, which corresponds to nothing known and seems very real, that is to say statistically significant. The only remaining hypothesis to explain the existence of these photons seeming to come from where there is the most dark matter, is that they would come from the disintegration of sterile neutrinos. As they are a little heavy, they would disintegrate producing "normal" neutrinos and photons whose energy would be half their mass. Abazajian considers that all dark matter consists of such $7 \mathrm{keV}$ sterile neutrinos.

\section{Heat, Entropy and Information Have Everything to Do with Black Holes}

\subsection{The Entropy Problem}

Entropy is forbidden to black holes by general relativity, because the theory requires them to be completely smooth, without substructure. General relativity describes a black hole as having a smooth geometry and indicates that every black hole of a given mass, spin and charge should be exactly the same: in other words, black holes have no hair.

In contrast, quantum mechanics says black holes have a large amount of entropy, meaning a microscopic structure, or a hair. In 1972, Jacob D. Bekenstein was the first to suggest that black holes should have a well-defined entropy. $\mathrm{He}$ wrote that a black hole's entropy was proportional to the area of its event horizon. Bekenstein also formulated the generalized second law of thermodynamics for systems including black holes. Both contributions were confirmed when Stephen Hawking (and, independently, Zeldovich and others) proposed the existence of radiation two years later. Hawking had initially opposed Bekenstein's idea on the grounds that a black hole could not radiate energy and therefore could not have entropy [29]. However, in 1974, Hawking performed a lengthy calculation that convinced him that particles can indeed be emitted from black holes. Today this is known as Hawking radiation.

Reflecting on the isolated black hole, Hawking noted that the light spectrum 
of the eponymous radiation streaming away from it would look the same as that of a radiating hot body, meaning that the black hole has a temperature. In general, temperature arises from the motion of atoms inside objects. The thermal nature of Hawking radiation, then, suggested that the black hole should have a microscopic structure made of some kind of discrete building blocks or bits. The work of Bekenstein and Hawking gives a formula for the number of bits, a measure known as the black hole entropy. Entropy is a gauge of disorder, which becomes greater as the number of states that an object can have grows. The larger the number of bits in a black hole, the more possible arrangements they can have and the greater the entropy.

So here is the contradiction: relativity says no hair, whereas quantum mechanics says black holes have a large amount of entropy, meaning some microscopic structure, or hair.

\subsection{The Information Paradox}

In agreement with the standard picture of quantum mechanics, information can never be destroyed. Even when you burn a letter, for example, the original information encoded in the atoms of the letter is preserved in the ashes. In quantum mechanics, every system is described by a formula called the wave function, which encodes the chances that the system will be in any particular state.

In keeping with Hawking's first calculation, the particles that escape from a black hole do not depend at all on the properties of the material that went into the hole. We could send a note with a message into the black hole, and there would then be no process to reconstruct the message from the final particles that would emerge. Hawking radiation implies that black holes destroy the information about the matter that falls into them. In Hawking's thought experiment, the loss of information means that we have no method to predict the wave function of Hawking radiation based on the properties of the mass that went into the black hole. Information loss is forbidden by quantum mechanics, so Hawking concludes that the laws of quantum physics had to be modified to allow for such loss in black holes.

In an effort to resolve these puzzles (this information paradox), physicists looked for new approach to combine general relativity and quantum mechanics into a coherent theory that could describe black holes. In 1997, Juan Maldacena came up with an idea around the information loss problem-a solution sometimes called the Maldacena duality. This duality is equivalence between quantum mechanics and gravity - a quantum theory of gravity. It means that the quantum physics of a black hole is equivalent to that of an ordinary gas of hot nuclear particles. It also means that spacetime is fundamentally different from what we perceive, more like a three-dimensional hologram projected from a two-dimensional surface of a sphere. If Maldacena's assumptions are true, then ordinary quantum laws would apply to gravity of black holes as well, and information cannot be lost [30]. 
Hawking had proposed that general relativity works for black holes but that quantum must be modified. Maldacena concludes that spacetime is holographic. In 2004 Hawking announced that he had changed his mind about the need for black holes to lose information.

\subsection{Entropy, Heat and Information of Black Hole According to the Theory of Relation}

We consider that quantum physics inside a black hole is equivalent to that of concentrated energy magma, or that of a gas of hot nuclear particles. According to the theory of the Relation, energy is "dark" for a double reason: it undergoes a change of energy (principle of Compensation) [31], and because a blackout accompanied by a charge reversal occurs at the passage of the event horizon.

We conjecture that energy within active black holes-surrounded by an accretion disk whose matter feeds them-is subject to high thermal quantum fluctuations (kinetic energy of particle motion). The temperature, that is to say the energy absorption capacity, is very high. Not only quantity of energy is huge but also its availability. According to quantum mechanics, pairs of particles and their antimatter counterparts are born incessantly, then disappear a few moments later in the universe. Pairs of real thermal particles that can be as well leptonic than bosonic. A huge amount of radiation and particles escapes from the inside of the black holes.

Under general relativity, no signal of any kind can come back from beyond the horizon because that would suppose exceed the speed of light. But if we rely on the equation that Hawking has derived from the temperature of a black hole [32],

$$
T^{o}=\frac{1}{16 \pi^{2}} \times \frac{c^{3} h}{G M k},
$$

( $T^{o}$ is temperature, $k$ is Boltzmann's constant, $k T^{o}$ is energy), it is not required that a particle exceeds the speed of light to cross the horizon to the outside. And there is no indication that the mass $M$ that melts contains only photons. It may contain hadrons. We stick to magnetic neutrinos.

In the expression

$$
k T^{o} M=\frac{1}{16 \pi^{2}} \times \frac{c^{3} h}{G},
$$

$T^{o}$ and $M$ are inversely proportional: when the temperature, or energy, increases, the classical mass declines. The contradiction between the diminishing mass and the growing energy seems flagrantly, since energy and mass are supposed to be equivalent. Clearly, the energy $k T^{o}$ contains the quantum mass $m_{o}$ which is proportional to the temperature. [ $m_{o}$ comes from $k T^{o}=h v=m_{o} c^{2}$. Equation (17) comes from $\left.t_{o} c=G M / c^{2}=h / m_{o} c=h / k T^{o}\right]$.

The link between energy, entropy and temperature refers to the second law of thermodynamics, which says that entropy always rises. The law of entropy implies irreversibility. The principle of irreversibility is that if you leave things to 
themselves at different temperatures, with the passage of time, their temperatures are getting closer and closer, and the availability of energy is continually decreasing. The one way always leads to a loss of energy availability. The drop in temperature, and therefore the decrease in the energy absorption capacity, goes hand in hand with an enlargement in entropy (which is a degraded energy) [33].

In the case of active black holes, there is a very high temperature around and beyond the horizon. The rise in temperature, and therefore the growth in energy, should go with a drop in entropy. But one concludes that the energy-mass increment goes hand in hand with a gain in entropy. In this case, the second law of thermodynamics, which states that entropy rises, presents a serious problem with temperature and energy [34].

Energy is a subtle concept, hard to grasp. It can be said that energy stops the motion as much as it provokes it. Bekenstein first conjectured that black holes have entropy. Entropy always goes hand in hand with energy. In itself, the existence of entropy does not imply that a system has a temperature. For Hawking, the key was temperature, not entropy. He anticipated that black holes also have a temperature. They are not cold objects, dead. They radiate thanks to an internal heat, but, in the end, it is this heat that causes their destruction.

On the subject of information, we think that the laws of general relativity are inapplicable beyond the horizon and that quantum mechanics must not be modified: information loss is forbidden. Imagine the particles falling into a black hole, each with its particular frequency that is its message. Very quickly, the sharply frequencies begin to dissolve, the message becomes almost impossible to discern in this magma of dark energy. The message becomes hopelessly scrambled in this inextricable mix of quantum fluctuations. The principles of quantum mechanics ensure that the message is always present within deformed particles moving in a chaotic manner. Although scrambled, not a single bit of information was eradicated. Each bit of information ends up being transferred to photons and other particles that evacuate energy from the black hole. The information is stored among the particles that form the Hawking radiation. The latter calculated that the disturbance of vacuum fluctuations due to black holes caused the emission of photons, as if the horizon of a black hole was a blackbody [32]. Hawking believed that a particle in a virtual pair escapes from the black hole but carries no information. Many theorists concluded that Hawking was wrong, that he had mistaken the scrambling of information for actual information loss. Our opinion is that not only information escapes from the black hole, but also the destroyed matter.

Perhaps the truth is somewhere in a hologram. A hologram is a two-dimensional image that makes it possible to reconstruct three-dimensional images. The holographic principle is a speculative conjecture in the framework of quantum gravity theory, proposed by Gerard't Hooft in 1993 and then improved by Leonard Susskind in 1995. This conjecture proposes that all the information contained in a volume of space can be described by a theory that lies 
on the edges of this region. For example, expanding cosmic space and black holes have horizons as an edge. The cosmic event horizon in an expanding universe is mathematically similar to the horizon of a black hole. The difference is that in the first case we are in and we look outward, and in the other we look at it from the outside. We can assume that the photons of the cosmic microwave background radiation that surround us are the messengers of the cosmic horizon that would carry the coded images of the megaverse. Just as one can surmise that the physical events that take place behind the horizon of the black hole would be telegraphed to the outside in a scrambled telegraph code in the form of Hawking radiation [35]. The idea that the universe is a kind of holographic image is surprising.

\section{Comments and Conclusions}

More than 80 percent of the mass of the universe is invisible. The presence of this dark matter is detected thanks to its gravitational signature [36]. His nature remains one of the great enigmas of cosmology. But we at least know that it is, for the most part, of a different nature from the ordinary matter that composes planets and stars [37]. In practice, the observations show that we cannot explain the distribution of matter by supposing that it is, on the one hand, solely baryonic and, on the other hand, governed only by the laws of gravitation. To reconcile theory and observation, scientists considered either changing the material content of the universe or changing the laws of gravitation. The hypothesis of an unknown form of matter remains the most accepted. A plethora of scenarios of high energy physics postulates new forms of matter that are difficult to detect [38]. Whether direct or indirect detection experiments, the tracks-especially the supersymmetric particle track-are very similar to a dead end. We will agree that in this moment of crisis, we must leave no track aside.

In this paper, we have just presented a radically different track to explain the enigma of dark matter: a major conceptual overhaul that concerns, in addition to dark matter, electromagnetism, sterile neutrino and black holes. Our model predicts that dark matter may be accompanied by a hidden and reworked version of electromagnetism (and possibly also a hidden weak force), implying that dark matter may emit and reflect hidden light. This "light" is invisible to us and so the dark matter remains unseen. Nevertheless, these new forces could have very significant effects. For example, they could distort interacting clouds of dark particles. Astronomers have sought this effect in the famous Bullet cluster, also called $1 \mathrm{E}$ 0657-56, which consists of two clusters of galaxies that have passed through each other. Observations show the co-mingling of clusters left the dark matter largely unperturbed, indicating that any dark forces are weak [39].

The new variant of electromagnetism, which we call magnetoelectricity, would also allow dark particles to exchange energy and momentum, a process that would tend to homogenize them and make the halos spherical. We can make 
some conclusions about the strength of the dark electromagnetism force-and thus how often dark matter annihilation occurs-by considering how this force would affect galaxies. The reason galaxies have a flattened structure is that electromagnetism allows ordinary matter to lose energy and settle into disks. Clouds of gas inside galaxies radiate electromagnetic energy through the emission of photons. That radiation results in the spinning matter inside the clouds clumping together and eventually relaxing into a dislike structure. Because we know that dark matter is primarily distributed spherically around most galaxies and does not collapse to a disk, we can conclude that it cannot lose energy via dark photon emission at the same rate that ordinary matter does [40].

We have seen above that if we apply the reversed laws of Maxwell and that if we try the same steps as those that led to the equation of the electron, we find another particle, no longer an electron but a magnetic monopole. A modification of the laws of gravitation in a somewhat ad hoc way constitutes an alternative to dark matter. Maxwell's reversed laws, on the contrary, justify the existence of this dark substance that would come from black holes.

Supermassive black holes are emerging as the most prolific creators. Far from being passive, they spit, blow. They emit large amounts of energy accumulated around them with unparalleled power. Their jets of matter would have fertilized the cosmos on vertiginous distances, triggered outbreaks of stars, created galaxies [2]. And why should not the supermassive black holes also have engendered dark matter? Why would not they also play the role of dark matter producer? Certainly not by condensed gas jets. Dark matter is different from ordinary matter, as is matter inside the black hole.

Our hypothesis of the inversion of Maxwell's laws as well as that of the black hole producing dark matter may seem as strange as absurd. But let us say it, the very existence of the dark matter seems absurd. Likewise the idea of black holes, which was originally a mathematical "catastrophe" shunned by theorists, including Einstein who had predicted them. Our view concerning the links between dark matter and black holes can be summarized as follows:

Dark matter is intimately related to black holes. The darkness of dark matter and black holes is caused by the reversal of Maxwell's laws. This inversion is triggered near the horizon of the black hole while the magnetic currents combined with gigantic pressure and high temperature cause a phase transition which results in a reversion of Maxwell's laws. This means that a magnetic charge is substituted for the electric charge, and that the magnetic current subdues the electric current. It can be said that in the space of the black hole a magnetoelectric force is created. The substance of dark matter comes from black holes. The latter emit particles from the process of creation of pairs of particles triggered by the metamorphosis of high energy photons. Growth of energy-mass of black holes increases the rate of materialization and annihilation of the dark energy inside. Dark radiation will materialize by creating a neutrino and an antineutrino, particles associated with the magnetic charge. If they are not annihi- 
lated, some will cross the black hole with a relativistic speed close to that of the light before slowing down to constitute the dark matter. But it can also happen that two opposite particles meet within the black hole. They dematerialize, they turn into two rays of the same energy and directed in the opposite direction. One of these dark rays, if not both, can cross the black hole without hitting an ordinary particle on the outside, and this dark radiation can materialize by creating a sterile magnetic neutrino and antineutrino accompanied by particles and by normal high energy photons. There is more than thermal evaporation; it is the spontaneous emission of particles. Black holes do not constitute dark matter, as we are led to believe. On the other hand, the black holes produce and emit the substance that constitutes the dark matter, in this case the sterile neutrino with magnetic charge. The black holes come undone, producing a dark matter that gradually disintegrates.

Crises in science are often the most creative. This redesign should have profound implications for theoretical physics and astrophysics.

\section{Conflicts of Interest}

The author declares no conflicts of interest regarding the publication of this paper.

\section{References}

[1] Rosenberg, L. (2018) Scientific American, 318, 53. https://doi.org/10.1038/scientificamerican0318-10

[2] Fontez, M., Ikonicoff, R., Grousson, M. and Benoît, R. (2018) Science \& Vie, No. 1204, 40-56.

[3] Bagdoo, R. (2019) Journal of Modern Physics, 10, 310-343. https://doi.org/10.4236/jmp.2019.103022

[4] Bagdoo, R. (2019) Journal of Modern Physics, 10, 922-952. https://doi.org/10.4236/jmp.2019.108061

[5] Gunzig, E. (2008) Quefaisiez-vousavant le big-bang? Odile Jacob, Paris, 163, 279-282, 287.

[6] Devos, S. (2019) Ciel \& Espace, No. 566, 42-61.

[7] Hutten, E.H. (1969) Les concepts de la physique. Dunod, Paris, 52.

[8] Dirac, P.A.M. (1931) Quantised Singularities in the Electromagnetic Field. Proceedings of the Royal Society of London. Series A, 133, 60-72. https://doi.org/10.1098/rspa.1931.0130

[9] Adair, R.K. (1987) The Great Design. Oxford University Press, New York, 134, 247.

[10] Song, J.S. (1996) The Journal of Undergraduate Sciences, 3, 47-55.

[11] Ikonicoff, R. and Benoît, R. (2019) Science \& Vie, No. 1224, 61-81.

[12] Thorne, K. (1994) Trous noirs et distorsions du temps. Champs, Flammarion, 466-7, 489, 496, 509-511, 515.

[13] Eisenstaedt, J. (2003) Einstein et la relativité générale. CNRS éditions, Paris, 432-486, 451, 460-1.

[14] Hawking, S. and Penrose, R. (1996) The Nature of Space and Time. Princeton University Press, Princeton, NJ, 75-77. 
[15] Price, R. and Thorne, K. (1997) Pour la Science. Dossier Hors-série juillet, 72-78.

[16] Hawking, S.W. (2014) Information Preservation and Weather Forecasting for Black Holes. arXiv:1401.5761v1.

[17] Bagdoo, R. (2019) Journal of Modern Physics, 10, 163-175. https://doi.org/10.4236/jmp.2019.102013

[18] Bertone, G. (2013) Le mystère de la matière noire. Dunod, 100-102+160-163.

[19] Magnan, C. (2011) Le théorème du jardin. amds Edition, 244+263.

[20] Bagdoo, R. (2011) Cosmological Inconstant, Supernova 1a and Decelerating Expansion. http://vixra.org/abs/1304.0169 https://www.academia.edu/5539777

[21] Colin, J., Mohayaee, R., Rameez, M. and Sarkar, S. (2019) Astronomy and Astrophysics, 631, L13. https://doi.org/10.1051/0004-6361/201936373

[22] Lochak, G. (2008) The Equation of a Light Leptonic Magnetic Monopole and Its Experimental Aspects. https://arxiv.org/ftp/arxiv/papers/0801/0801.2752.pdf

[23] Michaud, A. (2017) Electromagnetic Mechanics of Elementary Particles. 2nd Edition, Scholars' Press, Berlin, Chapter 9.

[24] Michaud, A. (2013) International Journal of Engineering Research and Development, 7, 50-66.

[25] Jeong, E.J. (2015) Physics of the Millennium: Birth of the New Paradigm. 26-132.

[26] Jeong, E.J. (1999) Physica Scripta, 59, 339-343. https://doi.org/10.1238/Physica.Regular.059a00339

[27] Abazajian, K. (2014) Physical Review Letters, 112, Article ID: 161303. https://doi.org/10.1103/PhysRevLett.112.161303

[28] Abazajian, K., et al. (2012) Light Sterile Neutrinos: A White Paper. http://arxiv.org/abs/1204.5379

[29] Overbye, D. (2015) Jacob Bekenstein. New York Times, B7.

[30] Polchinski, J. (2015) Scientific American, 312, 36-41. https://doi.org/10.1038/scientificamerican0415-36

[31] Bagdoo, R. (2008) The Pioneer Effect: A New Theory with a New Principle. http://vixra.org/abs/0812.0005 https://www.academia.edu/5535864

[32] Susskind, L. (2008) The Black Hole War. Back Bay Books, New York, 128-9, 132, 141-2, 148-9, 167-8, 172-3, 188-9.

[33] Feynman, R. (1980) La nature de la physique, Editions du Seuil, 144.

[34] Mayhew, K.W. (2018) New Thermodynamics: Say No to Entropy. 188.

[35] Susskind, L. (2007) Le paysage cosmique, folio essais. 514, 515, 518, 519.

[36] Bagdoo, R. (2012) Galaxy Rotation Curves Traced Out by the Theory of Relation. http://vixra.org/abs/1304.0079 https://www.academia.edu/5539791/

[37] Zweibel, E. (1998) Recherche. Hors-série avril, 10, 90.

[38] Riazuelo, A. (2011) Pour la Science. Dossier, No. 71, 66.

[39] Feng, J. and Trodden, M. (2014) Scientific American, 23, 44-51. https://doi.org/10.1038/scientificamericanuniverse0814-44

[40] Dobresco, B.A. and Lincoln, D. (2015) Scientific American, 313, 32-39. https://doi.org/10.1038/scientificamerican0715-32 